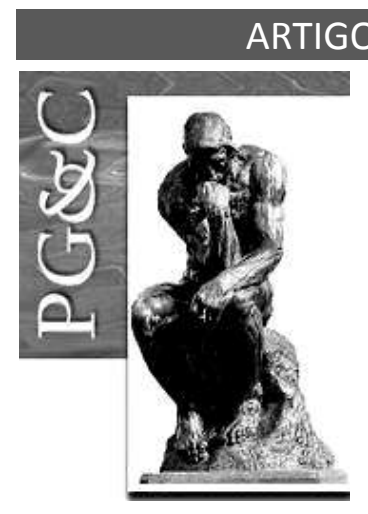

\title{
GESTÃO DO CONHECIMENTO TÁCITO NO CONTEXTO MIDDLE-UP-DOWN DA GESTÃO ESTRATÉGICA
}

\author{
Fernando Ramos Lengler \\ Doutorando em Administração pela Universidade do Estado de Santa \\ Catarina, Brasil. Professor do Complexo de Ensino Superior de Santa \\ Catarina, Brasil. \\ E-mail: fernandolengler@gmail.com
}

\author{
Amanda Santos Ilha \\ Mestranda em Design pela Universidade do Estado de Santa Catarina, Brasil. \\ E-mail: amandasilha@gmail.com \\ Rosalia Aldraci Barbosa Lavarda \\ Doutora em Administração pela Universitat de València, Espanha. \\ Professora da Universidade Federal de Santa Catarina, Brasil. \\ E-mail: rblavarda@gmail.com
}

\begin{abstract}
Resumo
Com o objetivo de compreender como se relacionam o conhecimento tácito e o processo integrador de formação da estratégia, este trabalho apresenta algumas reflexões decorrentes das mudanças nas organizações com foco na utilização da gestão estratégica middle-up-down, frente às condições dos ambientes internos e externos. É sabido que o conceito de estratégia e a gestão possuem diferentes correntes teóricas e não possuem um único direcionamento. Neste sentido, considerando-se a gestão do conhecimento e a implementação da estratégia propõe-se um ensaio teórico apresentando uma análise reflexiva sobre o processo de formação da estratégia middle-up-down, a partir das perspectivas de estratégias emergentes e deliberadas. Como principais reflexões, o ensaio destaca a constante influência das ações dos níveis intermediários das organizações e a troca de conhecimento organizacional pelos colaboradores de todos os níveis organizacionais na formação da estratégia. Destaca-se como contribuição deste estudo o indicativo para que as pesquisas realizadas dentro desta perspectiva aprofundem as teorias das ciências sociais contribuindo para a construção teórica do campo. Assim, a relação apresentada entre a gestão do conhecimento tácito (vivência organizacional) e o processo de formação da estratégia contribui para suscitar pesquisas que aprofundem o estudo das relações organizacionais e o que ocorre no dia a dia como algo de valor significativo para os estudos sobre estratégia.
\end{abstract}

Palavras-chave: Estratégia integradora. Middle-up-down. Gestão do Conhecimento. Conhecimento tácito. Ensaio teórico.

\section{TACIT KNOWLEDGE MANAGEMENT IN THE MIDDLE-UP-DOWN CONTEXT OF STRATEGIC MANAGEMENT}

\begin{abstract}
In order to understand how tacit knowledge and the strategy formation integrating process are related, this paper presents some reflections arising from the changes in organizations focusing on the use of strategic middle-up-down management, facing the internal and external environment conditions. It is well known that the concept of strategy and management have different theoretical currents and do not have single direction. In this sense, considering the knowledge management and the strategy implementation a theoretical essay was proposed, presenting a reflexive analysis about the middle-up-
\end{abstract}

Perspectivas em Gestão \& Conhecimento, João Pessoa, v. 10, n. 2, p. 3-21, maio/ago. 2020. DOI: http://dx.doi.org/10.21714/2236-417X2020v10n2p3

http://periodicos.ufpb.br/ojs2/index.php/pgc. ISSN: 2236-417X. Publicação sob Licença (cc) EY-NC-ND 
down strategy formation process, from the perspectives of emergent and deliberate strategies and the tacit knowledge in the context of strategic management. As main reflections, the essay emphasizes the constant influence of the actions of the intermediary levels of the organizations and the exchange of organizational knowledge by the collaborators of all the organizational levels in the strategy formation process. It is highlighted as contribution of this study the indicative so that the researches carried out within this perspective deepen the theories of the social sciences contributing to the theoretical construction of the field. Thus, the relationship between tacit knowledge management (organizational experience) and the strategy formation process contributes to research that deepens the study of organizational relationships and what occurs on a daily basis as something of significant value for strategy studies.

Keywords: Strategy; Integrative strategy. Middle-up-down. Tacit knowledge. Theoretical essay.

\section{INTRODUÇÃO}

É sabido que organizações de todos os tipos lutam para entender, adaptar e responder às mudanças nas condições dos ambientes internos e externos. Utilizam da gestão estratégica como ferramenta para atingir os objetivos almejados. Por várias décadas, o conceito de gestão estratégica foi construído por diferentes correntes teóricas, e ainda não há, na academia, uma definição universalmente aceita do termo e muito menos das práticas que o constituem.

Pressupõe-se que o processo de formação da estratégia aconteça de três formas distintas: deliberada (considerando-se a perspectiva de tomada de decisão top-down); emergente (a partir da tomada de decisão bottom-up) e/ou integradora (desde uma perspectiva de tomada de decisão middle-up-down) (ANDERSEN, 2004; LAVARDA; CANET; BONET, 2010; 2011).

Este estudo tem ênfase na abordagem processual da estratégia segundo o modelo de Mintzberg e Waters (1985) e Hart (1992) e no processo integrador de formação da estratégia (middle-up-down), (NONAKA, 1988; FLOYD; WOOLDRIDGE, 1992; FLOYD; LANE, 2000; ANDERSEN, 2000; 2004; 2013) que ainda têm limitado espaço nas mais conhecidas publicações do campo da estratégia quando comparado ao número de trabalhos que abordam fatores formais como planejamento e técnicas de análise. Poucos estudos se concentraram na relação entre a integração de diferentes modos e desempenho de estratégia (Hart, 1992; Hart e Banbury, 1994; Andersen, 2002).

Deste modo, são necessárias novas investigações sobre a interação entre planejamento e emergência no processo de formação de estratégias. Além disso, existe, por parte das organizações, um movimento que dá maior importância ao aproveitamento do capital intangível, principalmente na construção e planejamento das ações e estratégias. 0 capital intelectual e intangível das organizações é o conhecimento e a capacidade de aprendizado e, por esta questão, o termo gestão do conhecimento (GC), que faz a gestão deste capital, ganha relevância no meio empresarial e estratégico (STEWART, 1999; KLUGE, STEIN, LICHT, 2002; MARTINS, FERREIRA, 2015).

O conhecimento compartilhado corresponde ao cerne do desenvolvimento dos processos empresariais, sendo em grande parte responsável pela geração de diferenciais competitivos para organizações que buscam no Capital Intelectual as inovações capazes de agregar valor extraordinário (PRINZ; SYRI, 1998; QUINN, 1999; MILLS; SMITH, 2011; KIANTO et al., 2014; REZENDE et al., 2016).

Há um interesse crescente na complexidade da educação empresarial geral, bem como em disciplinas comerciais especializadas e no gerenciamento do conhecimento. A gestão do conhecimento nas organizações tem sido foco de investimentos crescentes, por meio de melhor capacitação e desenvolvimento do fator humano. Brandão e Guimarães (2011) 
abordam "a gestão de competências e a gestão de desempenho como instrumentos que fazem parte de um mesmo movimento, voltado a oferecer alternativas eficientes de gestão às organizações". Portanto, as políticas empresariais atuais buscam desenvolver suas competências, a fim de aumentar as respectivas chances de atingir seus objetivos estratégicos, via melhoria da qualidade nos processos de solução de problemas, negociações e de tomada de decisões.

Por outro lado, encontram-se estudos que apontam definições para o entendimento de conhecimento, classificando-o como tácito e explícito (NONAKA; TAKEUCHI, 1997); e, também, estudos sobre a gestão do conhecimento (NONAKA; KONNO, 1998). Ainda, teorias sobre o conhecimento baseado no indivíduo e no conhecimento organizacional (GARVIN, 1993), porém, fazendo uma busca no Spell (2018), um dos principais acervos de periódicos científicos eletrônicos brasileiro nota-se uma lacuna no estudo da relação do conhecimento tácito com o processo integrador de formação da estratégia.

Diante do exposto, o objetivo deste estudo foi compreender como se relacionam o conhecimento tácito e o processo integrador de formação da estratégia. Para tal optou-se por desenvolver um ensaio teórico (WHETTEN, 2003) cruzando estes dois campos do saber.

O trabalho se estrutura em três tópicos, além desta introdução. O segundo tópico apresenta uma revisão sobre estratégia, focada principalmente na abordagem processual e no processo integrador de formação de estratégia; seguido da Gestão do conhecimento e do Conhecimento tácito. O quarto tópico se refere ao conhecimento tácito no contexto do processo integrador de formação da estratégia e o quinto e último tópico se refere às considerações finais, seguidas das referências utilizadas na construção deste ensaio.

\section{ESTRATÉGIA: FORMAÇÃO E INTEGRAÇÃO}

Devido à complexidade inerente ao fenômeno e a diversidade de autores que escrevem sobre o tema, por várias décadas, o conceito de gestão estratégica foi construído por diferentes correntes teóricas, e ainda não há, na academia, uma definição universalmente aceita do termo e muito menos das práticas que o constituem (BULGACOV et al, 2007).

A origem grega da palavra estratégia indica objetivos claros, planejamento e comando. Os dicionários a descrevem como plano, método e estratagema (MARIOTTO, 2003).

Portanto, o termo estratégia não teve as suas origens no meio empresarial, mas advém da prática militar. Somente na década de 1950, foi incorporado aos negócios quando as empresas começavam a sentir a necessidade de preparação perante a concorrência. Era preciso uma visão de longo prazo, já que a sobrevivência não era mais garantida por objetivos fixados e ações de curto prazo (MOTTA, 1995).

Os primeiros estudos foram realizados por pesquisadores como Chandler (1962), Ansoff (1965) e Andrews (1971). Segundo Chandler (1962), estratégia é a definição dos objetivos básicos de longo prazo de uma empresa e a adoção das ações adequadas e de alocação dos recursos necessários para atingir tais objetivos. Para Ansoff (1965) a abordagem clássica de formação de estratégias, a do planejamento estratégico, defende que os objetivos gerais da organização são definidos pela alta direção, sendo depois desenvolvidos pela gerência de nível médio por meio de planos detalhados. Estes deverão ser validados pela alta direção antes que qualquer compromisso ou ação sejam realizados. Essa concepção de formação estratégica reflete uma abordagem de cima para baixo, onde os gerentes de nível médio têm espaço limitado para iniciativas estratégicas.

Mais tarde, Porter (1980) contribui para a definição de Chandler, em outros termos ele descreve a estratégia como meios definidos para obter os resultados organizacionais almejados.

Perspectivas em Gestão \& Conhecimento, João Pessoa, v. 10, n. 2, p. 3-21, maio./ago. 2020. 
Neste período, mais especificamente, no final da década de 1970, Mintzberg (1978) sentiu a necessidade de pensar a estratégia não em termos de intenções, mas sim em termos daquilo que a organização efetivamente faz, as ações que são realmente postas em prática e se dedicou à pesquisa do processo pelo qual as estratégias efetivamente se formam no mundo real das organizações, definindo estratégia como "um padrão em uma sucessão de decisões", (MINTZBERG, 1978), e, posteriormente, reformulou para "um padrão em uma sucessão de ações" (MINTZBERG; WATERS, 1985).

De acordo com Mintzberg e Waters (1985) a estratégia possui duas bases: uma deliberada e uma emergente e a organização trabalha dentro do processo de aprendizagem estratégica, na qual a retroalimentação traz ao processo o que emergiu no contexto.

Mintzberg (1978) definiu, já no início dos seus estudos que a estratégia deliberada é um padrão de ação seguido de acordo com um plano. É um processo planejado, consciente e explícito. Há na literatura inúmeras críticas às limitações das estratégias deliberadas, citando como exemplo, o fato de o processo desprezar a aprendizagem (MINTZBERG, 1978). As empresas comumente recorrem aos processos prescritivos de formulação estratégica - o chamado "planejamento estratégico", para encarar a incerteza ambiental e as questões que dizem respeito à sua sobrevivência. Acredita-se que a estratégia pode ser deliberada em um processo altamente formalizado e metodizado de concepção, levando a uma melhor adaptação aos fatores externos e organizacionais (MINTZBERG, 2004).

A outra base da estratégia, a estratégia emergente, também introduzida na década de 1970, por Henry Mintzberg (1978) já é comumente utilizada hoje na literatura de negócios e acadêmica, inclusive já há uma publicação acadêmica dedicada ao assunto, denominada Emergence: a journal of complexity issues in organizations and management. Outro importante acontecimento foi a fundação, em 1997, do Centro para Estratégia Emergente de Santa Fé (Novo México, EUA), que posteriormente passou a se chamar Santa Fe Associates International (MARIOTTO, 2003).

Na Figura 1 apresentam-se duas tarefas fundamentais do processo emergencial:

Figura 1 - Modelo simplificado de interpretação de estratégias emergentes

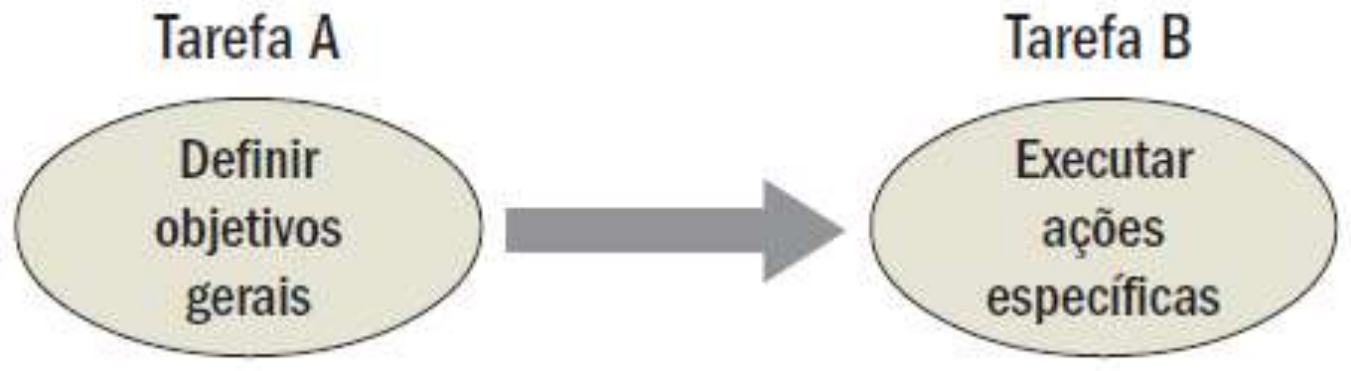

Fonte: Mariotto (2003, p.80)

Mariotto (2003) afirma que é comum uma divisão de trabalho para execução das tarefas A e B. Geralmente, a gerência de nível mais alto ou Top Management é responsável pela tarefa $A$ e os demais envolvidos, a gerência de nível mais baixo (Middle Management), operacional é responsável pela tarefa B.

Para uma estratégia ser deliberada pura, ela deve atender a três condições: primeira: devem existir intenções precisas, concretas, detalhadas na organização; segunda: as intenções devem ser virtualmente comuns a todos os atores organizacionais; terceira: as intenções devem ser realizadas exatamente como foram planejadas. Sendo assim, o ambiente externo deve ser perfeitamente previsível, totalmente benigno ou sob total controle da organização. 
Logo, para uma estratégia ser emergente pura deve haver ordem, consistente com a ação ao longo do tempo, na total ausência de intenção (MINTZBERG; AHLSTRAND; LAMPEL; 2010).

Para Mintzberg (1987, p. 66) na prática organizacional, a "formulação e implementação se unem em um processo fluido de aprendizado pelo qual as estratégias criativas se desenvolvem".

Complementando esta breve conceituação da abordagem processual, Mintzberg; Quinn (2001) definem o termo 'estratégia', como um padrão de decisões que determina e revela os objetivos, propósitos, políticas e planos de uma empresa. É essa estratégia que define a amplitude de negócios, a organização humana e econômica e as contribuições para proprietários, colaboradores, clientes e comunidades (MINTZBERG; QUINN, 2001), percebe-se que este é um conceito muito dinâmico e em constante reavaliação.

Essa diversidade de definições do termo estratégia é considerada por Mintzberg et al. (2007) a partir de cinco aspectos: (i) como um plano ou um conjunto de diretrizes para lidar com uma situação; (ii) pretexto, uma manobra para superar o concorrente; (iii) padrão de ações, que podem aparecer sem serem percebidos; (iv) posição (escolhida) ou um "nicho", domínio de determinado produto no mercado e (v) perspectiva, a forma como a organização vai agir, ligada à sua cultura e ideologias.

Além disso, há diversas tentativas de classificação da estratégia, podem-se destacar as dez escolas propostas por Mintzberg, Ahlstrand e Lampel (2010), as perspectivas genéricas (clássica, evolucionária, processualista e sistêmica) de Whittington (2002) e as perspectivas em gestão estratégica (clássica, moderna e pós-moderna) de Volberda (2004).

Após definir estratégia, Mintzberg et al (2010) também propôs o termo "formação de estratégia" para se referir ao processo pelo qual as estratégias são criadas; distinguindo de formulação como um processo criado por um grupo que não é o grupo que vai implementar. Este conceito de formação (distinto de formulação) não pretende distinguir quem formula e quem implementa a estratégia, passando esse a ser um processo contínuo.

Portanto, ao definir estratégia como um processo de formação, Mintzberg et al (2010) propõem que a separação clássica entre formulação e implementação deve ser eliminada, caracterizando a formação da estratégia como um processo contínuo de deliberação e emergência. Esta abordagem está alinhada à abordagem da estratégia como prática, que considera a formulação e a implementação como frequentes e inseparáveis (JOHNSON et al., 2003; REGNÉR, 2003, 2008; JARZABKOWSKI, 2005; WHITTINGTON, 2006; JARZABKOWSKI; BALOGUN; SEIDL, 2007).

Segundo Mariotto (2003) a pressão da concorrência é intensa, por isso as empresas precisam ter flexibilidade e inovação constantes. A forma clássica, periódica e formal de formação da estratégia não é mais suficiente para lidar com essa nova situação, que exige um processo contínuo, no qual as estratégias emergentes (juntamente com as estratégias deliberadas) podem ter um papel crucial.

Portanto, o processo estratégico vai além de um simples plano como foi inicialmente concebido. Estratégia envolve comportamento, ação, reflexão e padrões que emergem de forma incremental de uma coerência entre passado e futuro, pensar e agir, modelar e desenvolver. Essas relações se desenvolvem na sincronia do ambiente interno e externo que interagem e continuamente traçam o processo de mudança na alocação e no ordenamento de recursos, bem como na busca pelas vantagens competitivas da empresa (VALADÃO; SILVA, 2012).

Segundo Valadão e Silva (2012), as estratégias que fluem de forma emergente ou deliberada dentro da organização resultam de um processo de aprendizagem, no qual a análise e o amadurecimento possibilitam a identificação das incertezas do ambiente e permitem exercer alternativas viáveis para enfrentar o desconhecido, redesenhar o passado e dar amparo nas mudanças da empresa.

Perspectivas em Gestão \& Conhecimento, João Pessoa, v. 10, n. 2, p. 3-21, maio./ago. 2020. 
Esta pesquisa centra-se na abordagem integrativa, vista nos trabalhos de Mintzberg e Waters (1985), Hart (1992), Hart e Banbury (1994), Hamel e Prahalad (1994), Johnson et al. (2003), Andersen (2002, 2004, 2013), Elbanna (2006) e Lavarda; Canet-Giner; Peris-Bonet (2011).

Partindo do pressuposto de que é difícil imaginar uma ação com ausência total de intenção no ambiente organizacional, de tal forma que a estratégia puramente emergente é tão rara quanto a puramente deliberada (MINTZBERG, WATERS,1985), há de se considerar que o dia-a-dia organizacional é caracterizado pela forte presença do processo integrador de formação da estratégia que combina atividades descentralizadas (emergência) de tomada de decisão e planejamento central (deliberação) (BURGELMAN, 1983; MINTZBERG; AHLSTRAND; LAMPEL, 2000; MARIOTTO, 2003; CALDEIRA et al., 2009; LAVARDA; CANET-GINER; PERISBONET, 2010; 2011; OLIVEIRA et al., 2011, GABRICH; CASTRO, 2013).

Outra consideração relevante diz respeito ao ambiente de trabalho nas organizações necessitar de um processo de formação da estratégia onde exista a participação de todos os níveis hierárquicos, o que é a essência da perspectiva middle-up-down. Estrutura hierárquica, tanto de cima para baixo (top-down) como de baixo para cima (bottom-up) com gerentes de nível médio facilitadores de conhecimento (MINTZBERG; WATERS, 1985; NONAKA, 1988; HART, 1992).

A perspectiva middle-up-down é baseada na gestão de nível médio, com características empreendedoras e com um conhecimento, tanto explícito como tácito, e, ainda, com uma visão e objetivos comuns que orientem as atividades e ofereçam suporte para a tomada de decisão (NONAKA, 1988).

Andersen (2004) enfatiza que a formação de estratégia tem sido conceituada como cognição compartilhada entre os indivíduos que implementam a estratégia. $\mathrm{O}$ aprendizado contínuo das atividades organizacionais é um processo de aprendizagem social. A implicação dessas perspectivas é que as decisões estratégicas são influenciadas por gerentes de qualquer nível hierárquico e essa estratégia pode surgir ao longo do tempo como consequência das ações tomadas por esses decisores descentralizados. Por isso, Andersen (2004) define o emergencial estratégico descentralizado como decisões de compromisso de recursos realizadas por gerentes de nível inferior que posteriormente podem influenciar a direção estratégica da empresa.

Ao dar aos gerentes a autoridade para tomar decisões em áreas-chave quando as condições de concorrência mudam, as empresas tornam-se mais receptivas e alcançam melhores resultados, especialmente nos ambientes em constante mudança (ANDERSEN, 2004).

Diante do exposto, os processos estratégicos emergentes tornam-se mais importantes, pois aumentam a capacidade de resposta e adaptabilidade (BARTLETT; GHOSHAL, 1998; ANDERSEN, 2004). Ao mesmo tempo, o processo de planejamento estratégico pode apoiar a comunicação de insights tácitos de mercado que são essenciais para desenvolver estratégias eficazes (LORD; RANFT, 2000; ANDERSEN, 2004).

Os insights e o potencial da produção intelectual, produto do conhecimento, estão diretamente relacionados aos estímulos da gestão do conhecimento organizacional e acontecem com maior facilidade quando os colaboradores estão participando ativamente das atividades estratégicas, motivados e engajados com os objetivos organizacionais (NONAKA, 1988).

\section{GESTÃO DO CONHECIMENTO}

Cada vez mais há a necessidade por parte das empresas em valorizar o capital intangível, reflexo disso é a maior importância dada à marca, ao relacionamento com os 
clientes e aos colaboradores. Vinculado à identidade das empresas modernas, o capital intelectual e intangível é conhecido como o conhecimento e a capacidade de aprendizado potenciais dos colaboradores. Por isso, o termo gestão do conhecimento (GC) se alastra no meio empresarial, uma vez que faz a gestão deste capital. Então, a GC surgiu com a missão de repaginar o ambiente organizacional e trazer novos desafios (ROSA; TEIXEIRA; LAVARDA, 2012).

O conhecimento é um assunto muito estudado no campo da filosofia, desde o período grego até os dias atuais. A busca pela noção do termo "conhecimento" está associada à história da filosofia. Diversas definições são apontadas. A persistência do homem em estabelecer a ordem no caos leva-o a classificar tudo de acordo com as suas semelhanças, diferenças, periodicidade de tempo e causalidades, para poder agir sobre o ambiente e modificá-lo.

A proliferação de conceitos tais como os de "sociedade tecnológica" (por exemplo, BERGER et al. 1974), "sociedade de informação" (por exemplo, LYOTARD, 1984; BENIGER 1986), "sociedade de conhecimento" (BELL 1973, DRUCKER 1993, STEHR 1994), "sociedade de rico" ou "sociedade experimental" (BECK, 1992), dá corpo à visão (KNORR-CETINA, 1999) de um consenso no qual as sociedades ocidentais contemporâneas são governadas pelo conhecimento e pelo saber dos peritos.

Sob o olhar do conhecimento como prática social, Knorr-Cetina (1999) aborda os processos de produção de conhecimento de Culturas Epistêmicas - culturas que produzem e mantêm conhecimento científico. Essa abordagem está alinhada ao intuito deste estudo, visto que, o foco do seu interesse reside nos mecanismos de produção de conhecimento e não nos mecanismos de geração de produtos e artefatos (KNORR-CETINA, 1999). Produção esta que é desenvolvida por produtores de conhecimento, denominados cientistas.

Uma parte da bibliografia, no campo da sociologia da ciência, propõe uma abordagem que relaciona intimamente os mundos social e científico, remetendo-se, obrigatoriamente, à análise do relacionamento entre os cientistas, e destes com o mundo exterior, enfim, sobre qual é a base de organização. Introduzida por Thomas S. Kuhn a unidade produtora e legitimadora do conhecimento é chamada de comunidade científica (KUHN, 1978).

Diante do exposto, tem-se o conceito de conhecimento segundo Aranha e Martins (1993, p.21) como "o pensamento que resulta da relação que se estabelece entre o sujeito que conhece e o objeto a ser conhecido". Assim, para a apropriação intelectual do objeto é preciso que haja regularidade nos acontecimentos, caso contrário, não seria viável superar o estado de caos. O conhecimento se refere ao produto, ao resultado do conteúdo advindo do ato de conhecer, ou seja, o saber adquirido e acumulado pelo homem.

Do ponto de vista de Garcia (1988, p. 67), o conhecimento é "um produto do intelecto humano, voltado para o homem (...) que lhe permite entender, à sua maneira, o mundo que o cerca e, ao mesmo tempo, desenvolver técnicas para melhor viver nele". Segundo ele, conhecer significa descrever um fenômeno, sejam em suas particularidades estruturais, seja em seus aspectos funcionais; prever a probabilidade de ocorrência futura de um evento e, por fim, manipular e utilizar, um objeto qualquer, além de reproduzi-lo, alterando, até suas características básicas.

Nonaka e Takeuchi (1997) definem o conhecimento como "uma crença verdadeira justificada", corroborando com tal definição, Hessen (1999), afirma que a justificação visa a busca da verdade e a concordância do conteúdo do pensamento com o objeto, para certificação de que o conhecimento é verdadeiro.

O conhecimento pode ser classificado em sua dimensão epistemológica em conhecimento tácito e conhecimento explícito (NONAKA; TAKEUCHI, 1997). Este estudo dará ênfase ao conhecimento tácito, apresentando brevemente o conceito de conhecimento explícito, para efeito de diferenciação.

Perspectivas em Gestão \& Conhecimento, João Pessoa, v. 10, n. 2, p. 3-21, maio./ago. 2020. 
De forma geral, independentemente das diferentes abordagens, a expressão "conhecimento tácito" está relacionada ao conhecimento inato do ser humano, às habilidades adquiridas com a experiência, por isso é difícil de ser comunicado formalmente. A interação social é um requisito para que haja a experiência, e o conhecimento está nas pessoas, assim, é uma construção social. De nada adianta o indivíduo possuí-lo se não for socializado ou se não tiver aplicação prática relacionada ao grupo. Em outras palavras, se não for útil, o conhecimento tem pouco valor. O conhecimento tácito consiste nos modelos mentais, crenças e experiências que cada pessoa admite como certo (SENGE, 1990; MESO; SCHMITH, 2000), que podem ser expressos em atos e atitudes morais (POLANYI, 1962) e estão entranhados no indivíduo ou na organização (AMBROSINI; BOWMAN, 2001).

A visão de conhecimento construído como compreensão compartilhada que emerge do trabalho participativo e colaborativo inspira-se na construção conceitual de conhecimento tácito proposta por Michael Polanyi (POLANYI, 1962). Polanyi (1966) foi pioneiro na ideia de conhecimento tácito na ciência. Essa dimensão tácita do conhecimento, é difícil de ser explicitada, diz respeito a aquilo que nos possibilita "saber mais do que podemos dizer" (POLANYI, 1966, p. 4). Considera-se a condição do indivíduo como possuidor do conhecimento. As percepções são estimuladas por meio da interação das experiências passadas com a observação de algo exterior que lhe desperte a atenção.

Dessa maneira, a consciência do indivíduo vai se formando e, embora ele note as situações relacionadas a certos fenômenos, é incapaz de descrevê-los. Portanto, é notável a significativa relação estabelecida entre as experiências (práticas e/ ou intelectuais) do indivíduo e algo no mundo externo que lhe chame a atenção. No entanto, é necessário pontuar, que a capacidade de ver e sentir objetos externos pode ser adquirida pela experiência. É o aprender, fazendo (SOMENSI, 2005).

Polanyi (1966, p. 4) percebe a inteligência sob dois aspectos:

Inteligência-articulada e inteligência não-articulada. A inteligência nãoarticulada vem primeiro e a partilhamos com os animais. A inteligência articulada é reflexo da aquisição da linguagem, é própria do ser humano, o que o torna superior aos demais seres. A dimensão tácita do conhecimento é relacionada à inteligência não-articulada.

O conceito de Polanyi (1966) foi popularizado na área de GC por Nonaka e Takeuchi (1997), ao ressaltarem que as conclusões, insights e palpites subjetivos estão relacionados a esse tipo de conhecimento. Enquanto o conhecimento expresso vira informação, o conhecimento tácito é visto como único e capaz de gerar a inovação e, por isso, é mais valioso. Pode apresentar-se nos indivíduos, expressando-se, principalmente, por meio de habilidades, ou desenvolver-se em uma comunidade de prática especializada, onde recebe o nome de competência, capacitação ou know-how. O conhecimento tácito é prático, ou seja, segue um processo. Assim como, o know-how, que consiste em habilidades técnicas e/ou informais, capturadas em grupo e sistematicamente organizadas (NONAKA, 1991).

De acordo com Nonaka (1991), Ambrosini e Bowman (2001) o conhecimento tácito ocupa uma função central nas organizações do conhecimento que se sustentam ao longo do tempo. Para eles, o conhecimento é a base para a formação de competências e capacitações idiossincráticas, portanto, difíceis de imitar e transferir, uma vez que as principais características do conhecimento tácito afirmam que ele é pessoal, prático e específico ao contexto.

No contexto organizacional, o conhecimento tácito se apresenta como as habilidades individuais das pessoas que estabelecem as rotinas, que, por sua vez, servem como equivalentes metafóricos para o que se chama de "comportamento organizacional" (FOSS,

Perspectivas em Gestão \& Conhecimento, João Pessoa, v. 10, n. 2, p. 3-21, maio./ago. 2020. 
2003). Os conhecimentos tácitos e explícitos estão ativamente presentes nas organizações. Eles se complementam e a interação entre eles é a principal maneira de se criar conhecimento no âmbito organizacional, como relatam Santos (2001) e Carbone (2009).

De acordo com Fleury (2002, p.139):

É possível distinguir dois tipos de conhecimento: o explícito e o tácito. 0 conhecimento explícito, ou codificado, refere-se ao conhecimento transmissível em linguagem formal, sistemática, enquanto o conhecimento tácito possui uma qualidade pessoal, tornando-se mais difícil de ser formalizado e comunicado.

De acordo com Carbone (2009, p.82) o conhecimento tácito é "produzido pela experiência da vida, incluindo elementos cognitivos e práticos". Assim sendo, pode-se conceituá-lo como o conhecimento que é adquirido por meio de experiências individuais considerando-se fatores intangíveis, como por exemplo: crenças pessoais, ideias, valores, julgamentos pessoais, perspectivas, intuições.

Por sua natureza subjetiva é complicado formalizar o conhecimento tácito, pois "é dinâmico e somente pode ser acessado por meio de colaboração direta e de comunicação com pessoas que detém o conhecimento" como nos explica Lara (2004, p. 31). E é isso que torna o processo de transferência desse tipo de conhecimento nas empresas tão difícil, custoso e incerto, pois depende das experiências dos empregados. Mas se for bem explorado e essas experiências puderem ser transmitidas aos outros colaboradores, esse tipo de conhecimento torna-se um fator crítico de sucesso e fonte de competitividade no mercado.

De acordo com Lara (2004, p. 31), o conhecimento explícito "é adquirido principalmente pela educação formal e envolve conhecimento dos fatos". Assim sendo, podese conceituá-lo como o conhecimento que é tipicamente articulado na linguagem formal, como por exemplo: documentos, manuais, afirmações, especificações, multimídia.

Por sua natureza objetiva, o conhecimento explícito, portanto, é facilmente compartilhado entre os indivíduos, tornando mais simples o processo de disseminação do conhecimento na organização. Embora isso seja um ponto forte acaba se tornando uma ameaça, pois por sua facilidade de ser expresso, o conhecimento explícito corre o risco de ser comercializado e/ou revendido a concorrente por quem o adquire, como nos adverte Fleury (2002). Para reter o conhecimento no âmbito organizacional, deve-se patentear o capital intelectual.

Carbone (2009) e Nonaka e Takeuchi (1997) afirmam que os ocidentais tendem a dar maior ênfase ao conhecimento explícito por se relevar mais a importância do conhecimento formalizado. Em contrapartida, os japoneses tendem a dar maior ênfase ao conhecimento tácito por se relevar mais a importância do conhecimento como uma criação social. De um lado, a empresa é geralmente vista como uma máquina processadora de conhecimento, de outro, como um organismo vivo.

Diante disso, cabe às empresas que almejam ter sucesso, juntar o melhor dos dois mundos: "a atenção ao lado menos formal do conhecimento pelos japoneses, e a utilização das tecnologias da informação pelos ocidentais" como sugerem Nonaka e Takeuchi (1997, p. 286).

As empresas devem buscar o equilíbrio, pois a criação do conhecimento é proveniente da interação dos dois tipos de conhecimento, o tácito e o explícito. As empresas que conseguem alinhar e utilizar os conhecimentos existentes na organização tendem a se destacarem e serem empresas de sucesso.

Perspectivas em Gestão \& Conhecimento, João Pessoa, v. 10, n. 2, p. 3-21, maio./ago. 2020. 


\section{O CONHECIMENTO TÁCITO NO CONTEXTO DA ESTRATÉGIA INTEGRADORA}

Desenvolver a analogia de nível de organização com habilidades permite considerar que as organizações precisam das pessoas para desenvolver o conhecimento. São elas os atores neste processo. Além disso, a criação de um novo conhecimento não é um simples processamento de informações, mas depende de insights e intuições dos colaboradores para transformá-los em algo útil para o uso na organização (NONAKA, 1997). Essa "conversão do conhecimento", como é nomeado tal processo, é dinâmica e está ancorada na hipótese de que o conhecimento é criado e expandido por meio da interação social dos indivíduos ao lidar com o conhecimento tácito e explícito (NONAKA, TAKEUCHI, 1997). Andersen (2004) destaca que o aprendizado das atividades organizacionais é um processo de aprendizagem social.

O conhecimento tácito pode ser entendido como aquilo que um indivíduo é capaz de realizar com eficácia e que é adquirido por meio das experiências de vida deste indivíduo. Possui como características principais, ser pessoal e de difícil comunicação, por isso ele é dificilmente disseminado e imitado (POLANYI, 1966; NONAKA; TAKEUCHI, 1997; SOMENSI, 2005; ROSA et al, 2012).

Considerando tais características o conhecimento tácito é mais importante, diante de uma perspectiva organizacional (FOSS, 2003) e de grande valorização por organizações modernas, que consideram o capital humano, patrimônio (ROSA et al, 2012). Segundo Someni (2005), o conhecimento tácito é o elemento essencial para a gerar inovação nas organizações, sendo ele o grande potencial de criatividade e inovação. Isto se deve ao fato de que o conhecimento tácito pode ser um recurso que permite à organização desenvolver melhor que seus competidores suas habilidades diferenciais. Ele é heterogêneo, único, imperfeitamente móvel e difícil de copiar e não pode ser substituído (SOMENI, 2005).

Um processo integrador de formação da estratégia é mais eficiente adotando a perspectiva middle-up-down de tomada de decisão (NONAKA, 1988; MARTINS; LAVARDA, 2013), no qual há maior envolvimento dos gestores de nível médio (middle managers), já que eles estão no centro da gestão (entre deliberação e emergência) e, também, porque são os gestores intermediários que têm maior contato com todos os níveis organizacionais. Nonaka \& Takeuchi (1997, p.146), defendem que o conhecimento é criado pelos gerentes de nível médio, que são os líderes de forças tarefas, já que estão no centro do processo realizando a interseção dos fluxos de informações dos dois polos. São considerados como o "nó estratégico que liga a alta gerência aos gerentes da linha de frente", desempenhando um papel-chave na facilitação da criação do conhecimento organizacional.

No modelo middle-up-down, a alta gerência desenvolve uma visão ou sonho, enquanto os gerentes de nível médio criam conceitos mais concretos que os funcionários da linha de frente possam compreender e implementar. Todos devem participar da criação de conhecimento, mas há diferentes papéis nesse processo. Um dos papéis dos gerentes médio e seniores, por exemplo, é o de direcionar a confusão, que pode aparecer, para a criação do conhecimento. Confusão que pode ocorrer pois as pessoas não recebem um novo conhecimento de forma passiva, elas o recebem ativamente e adaptam às suas perspectivas. Assim, o que faz sentido em um contexto, pode mudar ou perder o sentido se comunicado a pessoas de um contexto distinto. Os gerentes são os responsáveis por direcionar esta confusão provendo os funcionários de uma estrutura conceitual que os ajude a dar sentido a sua própria existência. A seguir estão descritos os papéis de cada um, detalhadamente:

a) os funcionários da linha de frente (Profissionais do Conhecimento): sua função básica é a incorporação do conhecimento, tanto tácito quanto explícito. Os profissionais do conhecimento são classificados em dois grupos complementares: os operadores do conhecimento (geram e acumulam conhecimento tácito) e os especialistas do conhecimento (mobilizam o conhecimento explícito);

Perspectivas em Gestão \& Conhecimento, João Pessoa, v. 10, n. 2, p. 3-21, maio./ago. 2020. 
b) gerentes do nível médio (Engenheiros do Conhecimento): responsáveis pela facilitação do espiral do conhecimento abrangendo diferentes modos de conversão do conhecimento e diferentes níveis da organização;

c) alta gerência e gerentes seniores (Gerentes do Conhecimento): dão o senso de direção às atividades de criação do conhecimento de uma empresa, expressando os conceitos principais sobre o que a empresa deve ser, estabelecendo uma visão do conhecimento sob a forma de uma visão da empresa ou declaração de políticas e estabelecendo padrões que justifiquem o valor do conhecimento que está sendo criado (NONAKA; TAKEUCHI, 1997).

Nesse modelo, pode-se destacar a forte presença de uma unidade de análise em comum, tanto na perspectiva integradora, quanto no conhecimento tácito: a socialização. Segundo Nonaka e Takeuchi (1997), a socialização é um dos quatro modos de criação do conhecimento organizacional, denominados: socialização, externalização, combinação e internalização.

A socialização é o processo responsável pela transformação do conhecimento tácito em conhecimento tácito, por meio do compartilhamento de experiências, modelos mentais e habilidades técnicas. Um indivíduo pode adquirir conhecimento tácito de outros sem o uso da linguagem (NONAKA; TAKEUCHI, 1997).

A troca de experiências requer que os indivíduos envolvidos tenham algum tipo de experiência compartilhada em um mesmo contexto, como afirmam Nonaka e Takeuchi (1997, p. 69): "O segredo para a aquisição do conhecimento tácito é a experiência", o que vale dizer que, sem algum tipo de experiência será difícil alguém entender o raciocínio de outra pessoa. Os mestres trabalham com seus aprendizes e ensinam a eles sua arte, não por meio do discurso, mas sim pela observação, imitação e prática. Dito isso, sob a ótica da dimensão social do conhecimento, a interação humana tem importância estratégica, notadamente no compartilhamento do conhecimento tácito (SKYRME, 2000).

A confiança é um elemento indispensável na transferência de conhecimento entre indivíduos (ADLER, 2001), o que pode contribuir ou prejudicar o compartilhamento do conhecimento tácito (SNOWDEN, 1998). Há uma movimentação por parte das organizações para propiciar a interação e, assim, a troca de conhecimento.

As organizações modernas dispõem de ambientes projetados ou rodízio de colaboradores, fazendo com que estes se exponham ao conhecimento tácito de outros integrantes da equipe que até então não tinham contato. Wallace (2001) e Freitas e Leitão (2004) indicam o uso de equipes multifuncionais para otimizar a solução coletiva de problemas e reitera a importância dessa forma da comunicação face a face. A seriedade em transmitir uma visão do conhecimento deve ser a mesma de quando se propõem introduzir em toda a organização uma visão da missão e dos valores organizacionais. A mobilização de "ativistas" promove o engajamento, independentemente de nível hierárquico, na promoção e difusão do conhecimento. Globalizar o conhecimento local é fazer a difusão interativa do conhecimento ultrapassar barreiras geográficas.

É inadmissível, nos dias de hoje, considerar os colaboradores como meros fatores produtivos e mantê-los alheios ao processo; são eles os agentes transformadores da empresa e os responsáveis pela efetividade da organização (ROSA et al, 2012). Nesta difusão do conhecimento o que sobreleva é a comunicação humana, principalmente, na interação face a face. Interação esta, que acontece principalmente por meio dos gestores intermediários, visto que são eles que fazem o "meio-de-campo" entre as deliberações da alta gestão (top down) e a implementação (emergências) junto ao restante da organização (bottom-up).

De acordo com Nonaka (1988), os gerentes de nível médio, denominados profissionais do conhecimento, acumulam, geram e atualizam tanto o conhecimento tácito quanto o explícito, agindo como arquivos vivos no dia a dia da organização. Portanto, entende-se que o conhecimento tem influenciado as atuações dos gerentes de nível médio, pois ele é um dos

Perspectivas em Gestão \& Conhecimento, João Pessoa, v. 10, n. 2, p. 3-21, maio./ago. 2020. 
elementos responsáveis pela manutenção da estrutura hierárquica das empresas. Os gerentes de nível médio correspondem à categoria que de fato detêm o conhecimento e que dão sustentação aos estudos sobre modelo de tipologia de influência do middle manager de Floyd e Wooldridge (1992).

A organização que permite maior grau de participação está facilitando a melhoria de resultados organizacionais (LAVARDA et al., 2011). O processo integrador de formação da estratégia, que combina a racionalidade e a emergência (MINTZBERG; AHLSTRAND; LAMPEL, 2000; MARIOTTO, 2003; CALDEIRA et al., 2009; LAVARDA et al., 2011; OLIVEIRA et al., 2011), facilita uma gestão mais eficaz (LAVARDA et al, 2011; OLIVEIRA et al., 2011) e essa relação da perspectiva integrativa com o desempenho organizacional pode ser observado nos trabalhos de Andersen (2002, 2004).

Nessa linha argumentativa, pode-se dizer que a participação ativa de pessoas de todos os níveis organizacionais nas ações estratégicas é importante para a GC organizacional, pois este não ficará detido apenas à alta gestão (top managers) (MINTZBERG; WATERS, 1985; NONAKA, 1988; HART, 1992). Segundo Lavarda et al. (2011), sob a ótica da perspectiva micro organizacional, onde o desenvolvimento da tarefa ocorre, a organização pode se beneficiar, isso acontece porque mais pessoas e, mais frequentemente, estão envolvidas no processo de formação de estratégias, o que requer uma maior descentralização.

Andersen (2013) estabelece o conceito de formação estratégica descentralizada, no qual as decisões estratégicas são influenciadas por gerentes localizados em toda a organização e essa estratégia pode surgir ao longo do tempo como consequência das ações tomadas por esses decisores descentralizados. Ele ainda afirma que ao fornecer aos gerentes a autoridade para tomar decisões em áreas-chave, as empresas estarão mais preparadas às mudanças mercadológicas e provavelmente terão melhores resultados, especialmente nos ambientes em rápida mudança.

A constante busca por vantagens competitivas sinaliza a progressiva conviç̧ão de que entender o conhecimento é imprescindível para o sucesso e para a sobrevivência das organizações (DAVENPORT; PRUSAK, 1998).

Portanto, entende-se que a relação entre o conhecimento tácito e o processo integrador de formação da estratégia ocorre pela articulação proporcionada pelo nível médio da organização, responsável pela articulação entre o que é determinado e o que é efetivamente realizado. Também pode-se acrescentar que essa articulação e participação de todos os níveis organizacionais no processo de formação da estratégia é alvo do que tem sido designado como open strategy ou estratégia aberta, tema debatido no último European Group of Organization Studies (EGOS) (MACK; SZULANSKI, 2017) dentro do tema de interesse sobre estratégia como prática (HUTTER; NKETIA; FÜLLER, 2017; YAKIS-DOUGLAS et al., 2017).

\section{CONSIDERAÇÕES FINAIS}

Considerando-se o objetivo desta pesquisa de compreender como se relacionam o conhecimento tácito e o processo integrador de formação da estratégia, pode-se dizer que o mesmo foi alcançado, visto que se pode apresentar algumas reflexões pertinentes ao tema a partir deste ensaio teórico.

Quanto ao conhecimento tácito no contexto integrativo, observou-se que a relação acontece considerando os atores (pessoas/colaboradores) e o contexto (organizacional), principalmente por meio da socialização, modo de criação de conhecimento organizacional caracterizado pela troca de experiências (NONAKA; TAKEUCHI, 1997), a qual é fortemente presente na gestão intermediária (middle manager) (REGNÉR, 2003; LAVARDA; MACHADO, 2010; FRANZON et al., 2012), já que segundo Floyd e Wooldridge (1992), os gerentes de nível médio têm papéis estratégicos na estrutura da organização. 
O desempenho da organização é constantemente influenciado pelas ações do nível intermediário e, cada vez mais, a formação da estratégia é um assunto presente no cotidiano dos colaboradores de todos os níveis organizacionais, deixando de ser uma atividade restrita apenas à alta gestão (RÉGNER, 2003; LAVARDA; MACHADO, 2011).

Portanto, a otimização do processo de troca de conhecimento acontece quando este é descentralizado, propiciando a participação estratégica ativa de todos os níveis organizacionais (ANDERSEN, 2004), de forma a mantê-los motivados e engajados, como "ativistas" perante à troca de conhecimento organizacional (WALLACE, 2001; FREITAS; LEITÃO, 2004).

De forma sucinta, a GC é a união das informações que geram capital intelectual de todos os colaboradores de uma organização. Segundo Lara (2004), tem como objetivo prioritário fornecer ou aperfeiçoar a capacidade intelectual da empresa para os indivíduos que tomam as decisões no dia-a-dia organizacional que, em conjunto, determinam o sucesso ou o fracasso de um negócio. Portanto, a troca de conhecimento tácito é um diferencial competitivo a ser melhor explorado nas organizações modernas, que veem o capital humano como o maior ativo organizacional (LARA, 2004; ROSA; TEIXEIRA; LAVARDA, 2011).

Assim, entende-se que o conhecimento tácito proporcionado por uma formação estratégica mais aberta (middle-up-down) pode ser mais eficiente para responder aos desafios atuais.

A contribuição deste estudo está no ensejo de que as pesquisas realizadas dentro desta perspectiva aprofundem as teorias das ciências sociais contribuindo para a construção teórica do campo. Assim, a relação apresentada entre o conhecimento tácito (vivência organizacional) e o processo de formação da estratégia contribui para suscitar pesquisas que aprofundem o estudo das relações organizacionais e o que ocorre no dia-a-dia como algo de valor significativo para os estudos sobre estratégia.

As limitações do estudo correspondem a ser um trabalho teórico e não empírico, visto que não visa confirmações características de pesquisas empíricas. Os trabalhos teóricos, entretanto, possibilitam a reflexão e busca de novas linhas de pesquisa (WHETTEN, 2003).

Como futura linha de pesquisa pode-se considerar que a perspectiva de uma estratégia integradora que combina os planos deliberados com as ações emergentes reportando à importância de enxergar a forma como a estratégia é pensada e executada, os atores que fazem com que ela aconteça e a estratégia propriamente dita. Este pensamento implica em analisar a estratégia como prática, convencionalmente tratada como strategizing, a estratégia acontecendo. Além disso, é válido ressaltar a importante contribuição da gestão do conhecimento organizacional como facilitador da troca de conhecimento, principalmente tácito, na gestão do middle manager articulando a vivência organizacional como um valor estratégico.

Os estudos sobre a gestão do conhecimento tácito e como este se articula com o processo de implementação da estratégia middle-up-down está longe de ser esgotado, uma vez que as organizações demandam atualização constante dos estudos teóricos para melhor aprimorar suas práticas e, consequentemente, melhorar a qualidade de vida das pessoas dentro do ambiente organizacional.

\section{REFERÊNCIAS}

ADLER, P.S. Market, hierarchy, and trust: the knowledge economy and the future of capitalism. Organization Science, v.12, n. 2, p. 215-234, 2001.

AMBROSINI, V; BOWMAN, C. Tacit Knowledge: Some Suggestions for Operationalization. Journal of Management Studies, Oxford, v. 38, n. 6, p. 811-829, Set. 2001.

Perspectivas em Gestão \& Conhecimento, João Pessoa, v. 10, n. 2, p. 3-21, maio./ago. 2020. 
ANDERSEN, T. J. Strategic planning, autonomous actions and corporate performance. Long Range Planning, Vienna, v. 33, n. 2, p. 184-200, 2000.

ANDERSEN, T. J. How to reconcile the strategy dilemma? European Business Forum, v. 9, n. 1, p. 32-35, 2002.

ANDERSEN, T. J. Integrating the Strategy Formation Process: An International Perspective. European Management Journal, Glasgow, v. 22, n. 3, p. 263-272. 2004.

ANDERSEN, T. J. Short introduction to Strategic Management. Cambridge: University Printing House, 2013.

ANSOFF, H. I. Corporate strategy: an analytic approach to business policy for growth and expansion. New York: McGraw-Hill, 1965.

ARANHA, M. L.; MARTINS, M. H. Filosofando: introdução à filosofia. São Paulo: Moderna. 1993.

BARTLETT, C.A.; GHOSHAL, S. Beyond strategic planning to organizational learning: lifeblood of the individualized corporation. Strategy \& Leadership, Jan-Feb, p. 34-39, 1998.

BELL, D. The coming of post-industrial Society: a venture in social forecasting. New York: Basic Books, 1973

BECK, U. Risk Society: Towards a new modernity. London: Sage, 1992.

BENIGER, J. R. The control Revolution. Cambridge, MA: Harvard University Press, 1986

BERGER, P; BERGER, B; Kellner, H. The homeless mind: Modernization and Consciousness. New York: Vintage Books, 1974.

BRANDÃO, H., GUIMARÃES, T. Revista de Administração de Empresas - v.41, n. 1, p. 9, 2001.

BULGACOV, S. et al. Administração estratégica: teoria e prática. São Paulo: Atlas, 2007.

BURGELMAN, R. A. A model of the interaction of strategic behavior, corporate context, and the concept of strategy. Academy of Management Review, v. 8, n. 1, p. 61-70, 1983.

CARBONE, P. P. et al. Gestão por competências e gestão do conhecimento. 3. ed. Rio de Janeiro: FGV, 2009.

DAVENPORT, T.; PRUSAK, L. Conhecimento empresarial. Rio de Janeiro: Campus, 1998.

DRUCKER, P. Post-Capitalist Society. New York: Harper Collins, 1993.

ELBANNA, S. Strategic decision-making: Process perspectives. International Journal of Management Reviews, v. 8, n. 1, p. 1-20, 2006.

Perspectivas em Gestão \& Conhecimento, João Pessoa, v. 10, n. 2, p. 3-21, maio./ago. 2020. 
FLEURY, M. T. L.; OLIVEIRA Jr., M. M. Aprendizagem e gestão do conhecimento. In: FLEURY, Maria Tereza Leme (Coord.). As Pessoas na organização. São Paulo: Gente, 2002.

FLEURY, M. T. L. As pessoas na organização. São Paulo: Gente, 2002.

FLOYD, S. W. \& WOOLDRIDGE, B. Middle management involvement in strategy and its association with strategic type: A research note. Strategic Management Journal, v. 34, n. 13, p. 153-167, 1992.

FOSS, N. J. Bouded rationality and tacit knowledge in the organizational capabilities approach: An Assessment and a re-evaluation. Industrial and Corporate Change, Oxford, v. 12, n. 2, p. 185-194, April 2003.

FRANZON, F.; OLIVEIRA, D.; LAVARDA, R. A. B. Atuação do gerente intermediário no processo de formulação e implantação da estratégia: um estudo de caso. Revista Brasileira de Administração Científica, Aquidabã, v.3, n.3, p.128-146, 2012.

FREITAS, J. A. S. B.; LEITÃO, S. P. Em busca de uma abordagem integrativa do conhecimento organizacional. Revista Organizações e Sociedade. v. 11, n. 30, maio/ago, p. 13-28, 2004.

GARCIA, F. L. Introdução Crítica ao Conhecimento. Campinas: Papirus, 1988. 113 p.

GABRICH, R.; CASTRO, J.M. A combinação entre estratégias deliberadas e emergentes em organizações: estudo de casos comparativos em ambientes dinâmicos. Contextus: Revista Contemporânea de Economia e Gestão, Fortaleza, v. 11, n. 1, p. 66-85, jan./jun. 2013.

HART, S. L. An integrative framework for strategy-making processes. Academy of Management Review, v. 17, n. 2, p. 327-351, 1992.

HART, S.; BANBURY, C. How strategy-making processes can make a difference. Strategic Management Journal, v. 15, n. 4, p. 251-269, 1994.

HESSEN, J. Teoria do Conhecimento. Tradução de João Vergílio Gallerani Cuter; revisão técnica Sérgio Sérvulo da Cunha. São Paulo: Martins Fontes, 1999. Título original: Erkenntnostheorie.

HUTTER, K.; NKETIA, B. A.; FÜLLER, J. Falling short with participation-different effects of ideation, commenting, and evaluating behavior on open strategizing. Long Range Planning, $\mathrm{v}$. 50, n. 3, p. 355-370, 2017.

JARZABKOWSKI, P. Strategy as practice: an activity-based approach. London: Sage, 2005.

JARZABKOWSKI, P.; BALOGUN, J.; SEIDL, D. Strategizing: the challenge of a practice perspective. Human Relation, v. 60, n. 1, p. 5-27, 2007.

JOHNSON, G.; MELIN, L.; WHITTINGTON, R. Micro Strategy and Strategizing: Towards an Activity-Based View. Journal of Management Studies, v. 40, n. 1, p. 3-22, 2003.

KIANTO, Aino et al. The interaction of intellectual capital assets and knowledge management practices in organizational value creation. Journal of Intellectual Capital, v. 15, n. 3, p. 362375, 2014.

Perspectivas em Gestão \& Conhecimento, João Pessoa, v. 10, n. 2, p. 3-21, maio./ago. 2020. 
KLUGE, J.; STEIN, W.; LICHT, T. Gestão do conhecimento: segundo um estudo da McKinsey \& Company. Lisboa: Principia, 2002.

KNORR-CETINA, K. Epistemic Cultures: How the Sciences Makes Knowledge. Cambridge: Harvard University Press, 1999.

KUHN, T. A Estrutura das Revoluções Científicas. 2. ed. São Paulo: Editora Perspectiva, 1978.

LAVARDA, R. B.; MACHADO, J. A. A influência do middle manager na formulação e implementação da estratégia de uma instituição de ensino superior privada em Boa Vista-RR. In: SEMEAD, 13., São Paulo, 13, 2010, p. 2177- 3866, 2010.

LAVARDA, R. B.; CANET-GINER, M. T.; PERIS-BONET, F. J. How Middle Managers Contribute to Strategy Formation Process: Connection of Strategy Processes and Strategy Practices. Revista de Administração de Empresas, São Paulo, v. 50, n. 4, 2010.

LAVARDA, R. B.; CANET-GINER, M. T.; PERIS-BONET, F. J. Understanding how the strategy formation process interacts with the management of complex work. European Business Review, v. 23 n. 1, p. 71-86, 2011.

LARA, C. R. D. A atual gestão do conhecimento: a importância de avaliar e identificar o capital intelectual nas organizações. São Paulo: Nobel, 2004.

LORD, M. D.; RANFT, A.L Organizational learning about new international markets: exploring the internal transfer of local market knowledge. Journal of International Business Studies, v.31, n. 4, p. 573-589, 2000.

LYOTARD, J. F. The postmodern condition. Minneapolis: University of Minnesota Press, 1984.

MACK, D. Z.; SZULANSKI, G. Opening up: how centralization affects participation and inclusion in strategy making. Long Range Planning, v. 50, n. 3, p. 385-396, 2017.

MARIOTTO, F. L. Mobilizando Estratégias Emergentes. RAE-revista de administração de empresas, v. 43, n. 2, p. 78-93, 2003.

MARTINS, G. T.; LAVARDA, R. B. Modelo integrador de formação da estratégia: um estudo de caso. Revista Organizações em Contexto, v. 9, n. 17, jan/jun, p. 125-153, 2013.

MARTINS, H. F.; FERREIRA, A. C. Capital Intelectual e o Ensino Superior: Análise e Perspectivas. Perspectivas em Gestão \& Conhecimento, João Pessoa, v. 5, n. 2, p. 83-110, jul./dez. 2015.

MATIAS-PEREIRA, J. Manual de Metodologia da Pesquisa Científica. São Paulo: Atlas, 2007

MESO, P; SMITH, R. A Resource-Based View of Organizational Knowledge Management Systems. Journal of Knowledge Managemet, Kempston, v. 4, n. 3, p. 224-231, 2000.

MINTZBERG, H. Patterns in strategy formation. Management Science, v. 24, n. 9, p. 934-948, 1978. 
MINTZBERG, H. Crafting Strategy. Harvard Business Review, v. 57, n. 4, p. 65-75, 1987

MINTZBERG, H. Ascensão e queda do planejamento estratégico. Porto Alegre: Bookman, 2004.

MINTZBERG, H.; WATERS, J. A. Of strategies, deliberate and emergent. Strategic Management Journal, Jul-Sep, 6, 3: 257-272, 1985.

MINTZBERG, H.; QUINN, J. B. O processo da estratégia. 3. ed. Porto Alegre: Bookman, 2001.

MINTZBERG, $\mathrm{H}$. et al. $\mathrm{O}$ processo da estratégia: conceitos, contextos e casos selecionados. Tradução Luciana de Oliveira da Rocha. Porto Alegre: Artmed, 2007.

MINTZBERG, H.; AHLSTRAND, B.; LAMPEL, J. Safari de estratégia: um roteiro pela selva do planejamento estratégico (2a ed.). Porto Alegre: Bookman, 2010.

MOTTA, P. R. A ciência e a arte de ser dirigente. 6. ed. Rio de Janeiro: Record, 1995.

MOTTA-ROTH, D.; HENDGES, G. R. Produção textual na universidade. São Paulo: Parábola, 2010.

MILLS, Annette M.; SMITH, Trevor A. Knowledge management and organizational performance: a decomposed view. Journal of Knowledge Management, v. 15, n. 1, p. 156-171, 2011.

NONAKA, I. Toward middle-up-down management: accelerating information creation. Sloan Management Review, v. 29, n. 3, p. 9-18, 1988.

NONAKA, I. TAKEUCHI, H. Criação de conhecimento na empresa: como as empresas japonesas geram a dinâmica da inovação. Rio de Janeiro: Elsevier, 1997.

NONAKA, I.; KONNO, N.; The Concept of "Ba": Building a Fuoundation for Knowledge Creation; California Management Review, v. 40, n. 3, p. 40 - 54; 1998.

POLANYI, M. Personal knowledge: towards a post-critical philosophy. Chicago: The University of Chicago Press, Corrected edition, 1962.

POLANYI, M. The Tacit Dimension. New York: Doubleday \& Company, Inc., 1983. 108 p. Trabalho original publicado em 1966.

POLANYI, M. The Tacit Dimension. New York: Doubleday \& Company, Inc., 1983. 108 p. Trabalho original publicado em 1966.

PORTER, M. Estratégia Competitiva: Técnicas para Análise da Indústria e da Concorrência. Ed. Campus, 1980.

PRINZ, W.; SYRI, A. An environment for cooperative knowledge processing. In: Information Technology for Knowledge Management. Springer Berlin Heidelberg, 1998.

Perspectivas em Gestão \& Conhecimento, João Pessoa, v. 10, n. 2, p. 3-21, maio./ago. 2020. 
QUINN, J. B. Strategic outsourcing: leveraging knowledge capabilities. MIT Sloan Management Review, v. 40, n. 4, p. 9, 1999.

REGNÉR, P. Strategy Creation in the Periphery: Inductive Versus Deductive Strategy Making, Journal of Management Studies, v. 40, n. 1, p. 57-82, 2003.

REGNÉR, P. Strategy-as-practice and dynamic capabilities: steps towards a dynamic view of strategy. Human Relations, v. 61, p. 565-588, 2008.

REZENDE et al., 2016. Plataformas para gestão do conhecimento: Estudo de caso sobre a ativação do valor de excedentes cognitivos por meio do desenvolvimento de um contexto capacitante virtual. Perspectivas em Gestão \& Conhecimento, João Pessoa, v. 6, n. 1, p. 72-88, jan./jun. 2016.

ROSA, C. P.; TEIXEIRA, J. E. C.; LAVARDA, R. B. A influência do conhecimento na atuação dos gerentes de nível médio no processo de formação da estratégia. Revista Ciências Administrativas (UNIFOR), v. 17, p. 621-649, 2012.

SANTOS, A. R. Gestão do conhecimento: uma experiência para o sucesso empresarial. Curitiba: Champagnat, 2001.

SENGE, Peter M. A Quinta Disciplina: Arte, Teoria e Prática da Organização de Aprendizagem. Trad. de Regina Amarante. 12 ed. São Paulo: Ed. Nova Cultura Ltda, 1990. 352 p. Título original: The Fifth Discipline. STEHR, N. Arbeit, Eigentum und Wissen: Zur theorie von Wissensgesellschaften. Frankfurt am Main: Suhrkamp, 1994.

SKYRME, D. J. Developing a knowledge strategy: from management to leadership. In: MOREY, D.; MAYBURY, M.; THURAISINGHAM, B. (ed.). Knowledg management: classic and contemporary works. Cambridge, MS: MIT Press, 2000.

SNOWDEN, D. The ecology of a sustainable knowledge program. Knowledge Management, v.1, n. 6, p.1-14, 1998.

SOMENSI, N. F. Variáveis que influenciam o desenvolvimento do conhecimento no curso de mestrado, na percepção dos egressos. 2005. 136 f. Dissertação (Mestrado em Administração) - Curso de Pós-Graduação em Administração. Universidade Federal de Santa Catarina, Florianópolis, 2005.

SPELL. SPELL ${ }^{\circledR}$ Scientific Periodicals Electronic Library. Disponível em: http://www.spell.org.br/. Acesso em: 02 fev. 2018.

STEWART, T. Capital Intelectual: a nova riqueza das organizações. Lisboa: Edições Sílabo, 1999.

VALADÃO, J. A. D.; SILVA, S. S. S. Justaposições da estratégia como prática e processo de estratégia: antes da visão pós-processual da estratégia. Revista de Administração Mackenzie, v. 13, n. 2, p. 171-195, Mar./Abr. 2012.

VOLBERDA, H. W. Crisis in Strategy: fragmentation, integration or synthesis. European Management Review, v. 1, n. 1, p. 35-42, 2004.

Perspectivas em Gestão \& Conhecimento, João Pessoa, v. 10, n. 2, p. 3-21, maio./ago. 2020. 
VOLBERDA, H. W. Crise em estratégia: fragmentação, integração ou síntese. Revista de Administração de Empresas, v. 44, n. 4, p. 32-43, 2004.

WALLACE, W. Knowledge creation and innovation: meeting the tacit knowledge challenge. In: KNOWLEDGE MANAGEMENT \& ORGANIZATIONAL LEARNING CONFERENCE, $3^{\text {rd }}$., Proceedings [...]. London, March 12-15, 2001.

WHETTEN, D. A. Desenvolvimento de teoria. O que constitui uma contribuição teórica? RAErevista de administração de empresas, v. 43, n. 3, p. 69-73, 2003.

WHITTINGTON, R. O que é estratégia. São Paulo: Pioneira, 2002.

YAKIS-DOUGLAS, B. et al. Opening M\&A strategy to investors: predictors and outcomes of transparency during organizational transition. Long Range Planning, v. 50, n. 3, p. 411-422, 2017.

Artigo recebido em 13/06/2018 e aceito para publicação em 02/04/2020 